\title{
Editorial
}

\section{Diabetic emergencies and the Loch Ness monster}

\author{
L. Axelrod \\ The Diabetes Unit, Massachusetts General Hospital, Boston, Massachusetts, USA
}

The fluid and electrolyte deficits of diabetic ketoacidosis (DKA) arise when severe insulin deficiency exists for a protracted period of time, typically several days. During the genesis of DKA, the patient develops profound losses of water and electrolytes, including not only sodium and chloride, but also potassium, phosphate and magnesium. These losses are largely but not entirely the consequence of an osmotic diuresis. As with any osmotic diuresis, the loss of free water exceeds the loss of electrolytes. Consequently, the patient develops a state of hypertonic dehydration, with both intracellular and extracellular dehydration. Other factors that contribute to the fluid and electrolyte losses include vomiting, ketonuria (with obligatory losses of cations to accompany the ketones) and decreased renal tubular reabsorption of sodium. (Insulin promotes sodium reabsorption at the distal tubule.) The pathogenesis of the fluid and electrolyte deficiencies of hyperosmolar nonketotic coma (HNKC) is similar, except that the fluid losses are more profound and ketonuria does not contribute.

In general, the managment of DKA and HNKC involves the administration of insulin in sufficient quantity, the repletion of the fluid and electrolyte (sodium, chloride and potassium) losses, and the treatment of any associated illness, which may have precipitated or exacerbated the acute episode [1].

Regimens for the replacement of fluid and electrolytes, the principal concern of this commentary, evolved from knowledge of the losses that occur during the genesis of DKA and HNKC. Simply stated, physicians have attempted to replace what is lost. If one were to consider fluid and electrolyte management of DKA simply as a matter of repletion of losses, then one would use hypotonic fluids such as half normal saline. But it is not that simple. Some but not all patients with DKA and HNKC are hypotensive at the time of presentation. Furthermore, administration of insulin - an essential part of the management of DKA or HNKC in every patient - may exacerbate hypotension as a consequence of the insulin-mediated transport of glucose from the extracellular fluid space to the intracellular fluid space and the ensuing osmotic movement of water from the extracellular fluid space to the intracellular fluid space. In other words, things may get worse before they get better. Consequently, the physician must defend the patient's blood pressure as well as the osmolality. The body defends blood pressure over tonicity, as in the appropriate release of antidiuretic hormone in the patient with volume depletion without hyperosmolality. So, too, the physician must give priority to the preservation of blood pressure before correcting hyperosmolality.

Thus, many physicians initiate therapy in a diabetic emergency with isotonic fluid until the patient's hemodynamic stability is assured and then change to hypotonic fluid replacement, typically after 1 or $2 \mathrm{~h}$, to correct the patient's deficits properly. This is my own preference. Others use the estimated or measured serum osmolality [2] or serum sodium [1] before therapy as a guide to the choice between hypotonic or isotonic fluid replacement. These physicians begin with isotonic fluid unless the serum osmolality or the serum sodium exceeds an arbitrary level, e.g. an osmolality above $320 \mathrm{mOsm} / \mathrm{Kg}$ or a sodium above $150 \mathrm{mEq} / 1$. If so, they use hypotonic fluids. Although these approaches are reasonable on theoretical grounds and are typical of current management they are not supported by data from a prospective, controlled clinical study in which initial therapy with isotonic fluid followed by hypotonic fluid is compared with hypotonic fluid alone. As elsewhere in clinical practice, controversy thrives on the absence of information. Previous controversies in the management of DKA, such as the proper dose of insulin or the possi- 
ble role of phosphate replacement, have only been settled by prospective, controlled studies.

This unsettled state of affairs is further complicated by the fear that repletion of fluid losses may actually be harmful to patients with DKA. Specifically, concern arises that current therapy of DKA may be associated with cerebral edema, noncardiogenic pulmonary edema and interstitial edema. The spectre of these edematous states as an important clinical problem in DKA shares certain attributes with the Loch Ness monster [3]. In both instances, the concern is based more on strongly held belief than on objective data. Like Nessie, the metabolic spectre returns over and over again. Unfortunately, the analogy rapidly breaks down. Little harm is done by chasing the Loch Ness monster, but great harm may result from pursuit of the metabolic monster [3].

Although patients with DKA frequently have subclinical evidence of cerebral edema or pulmonary edema [4-6], clinically evident cerebral edema and pulmonary edema are rare in DKA [3-7]. Of course, the rare case of cerebral or pulmonary edema in DKA may represent an extension of the subclinical cerebral or pulmonary edema that is commonly seen in DKA. This remains to be proven $[5,6]$. It is also possible that the occasional association of clinically evident cerebral edema or pulmonary edema with DKA is due to the chance intercurrence of two unrelated conditions. The available data do not exclude this possibility. In fact, in one report where pulmonary edema was attributed to crystalloid infusion in two patients both patients had a history of alcohol abuse and hypoalbuminemia [8]. In another case report, the patient also had hypoalbuminemia, the cause of which was not stated [9]. Surprisingly, most patients with noncardiogenic pulmonary edema (acute respiratory distress syndrome, ARDS) associated with uncontrolled diabetes are under 50 years old and have severe acidosis; this complication is relatively rare in older patients with HNKC [7]. This suggests that the pathophysiology is more closely related to the pathogenesis of the ketotic state than to the hyperosmolality or its correction. Plasma free fatty acids, which are invariably elevated in DKA but are typically not elevated in HNKC, may contribute to some of the complications of acute pancreatitis, including ARDS [10]. Possibly, the elevated plasma free fatty acid levels in DKA may have a similar effect in this disorder. Similarly, arachidonic acid may induce brain swelling by a toxic effect on the cerebral capillary endothelium, the locus of the blood brain barrier [6]. Anoxia and changes in the oxygen dissociation curve are other possible explanations.

Nor is there any satisfactory evidence that aggressive treatment with crystalloids will precipitate clin- ically evident cerebral or pulmonary edema in DKA. On the contrary, aggressive management with hypotonic (half-normal) crystalloid solutions at rates up to $2 \mathrm{l} / \mathrm{h}$ has not been associated with fatal cerebral edema [7]. In fact, inadequate replacement with crystalloid in DKA and HNKC is probably a more frequent and serious problem than overtreatment $[2,3]$. In short, the role of crystalloid therapy in the genesis of the cerebral and pulmonary edema of uncontrolled diabetes is not proven and alternative explanations exist.

In the current issue of Intensive Care Medicine, Hillman proposes the use of colloid in the management of "all diabetic emergencies who present with shock" due to hypovolemia. This is based on the rationale that isotonic saline is inefficient (and hypotonic saline is even more inefficient) in restoring circulating volume because it is mainly distributed to the interstitial space and because overexpansion of the interstitial space may be deleterious, i. e. may result in cerebral, pulmonary and peripheral edema.

One must ask, first of all, which patients Hillman proposes to treat with colloid. Does he mean to suggest that all patients with a diabetic emergency are in shock? Although he addresses his comments to all diabetic emergencies who present with shock due to hypovolemia the rationale is applied more broadly and shock is not defined. Although patients with DKA and hyperosmolar nonketotic coma (HNKC) are invariably hypovolemic only a small fraction are in shock, if that is defined as a systolic blood pressure below $90 \mathrm{~mm} \mathrm{Hg}$. Especially in DKA, patients are often surprisingly warm and well perfused despite the hypovolemia. The majority of patients with DKA and HNKC respond well to crystalloid replacement.

Secondly, there is no direct evidence that colloid is preferable to crystalloid in patients with DKA or HNKC. This hypothesis has not been tested. At best, the rationale for colloid is a justification for a controlled study. There are no data to support the use of colloid routinely in the treatment of DKA or HNKC. The argument that colloid is preferable to crystalloid in other circumstances is not compelling. For one thing, argument by analogy is not a strong form of argument. For another, careful studies indicate that the use of colloid is not preferable to crystalloid in many circumstances. This was the experience of military surgeons with battlefield injuries during the war in Vietnam. Controlled studies indicate that colloid is not preferable to crystalloid in hypovolemic shock [11], abdominal aortic reconstructive surgery $[12,13]$, laparotomy for trauma [14], pulmonary insufficiency [15] and thermal injury [16].

Thirdly, the fear of crystalloid in diabetic emergencies is not justified, as noted above. Undertreatment may be a more serious problem than overtreatment. 
Fourthly, colloid is not without risk. Dr. Hillman does not specify which form of colloid he has in mind. Presumably he would use albumin, since it does not carry an appreciable risk of transmission of viral hepatitis or acquired immunodeficiency syndrome (AIDS). Nevertheless, even albumin carries a low but appreciable risk of anaphylaxis, perhaps one in 200,000 cases. Synthetic colloid solutions such as dextran or hydroxyethylstarch carry a higher risk of anaphylaxis. Certainly, the advice to use colloid should not be construed to justify the use of fresh frozen plasma, which can transmit viral hepatitis or AIDS, in diabetic emergencies.

Finally, colloid is expensive and should not be used unless the benefits justify the cost.

In summary, the rationale for the use of colloid in patients with DKA and HNKC is not supported (or refuted) by any controlled clinical trial. The rationale, based on the alleged inefficiency of crystalloid in the restoration of circulating volume and the possible deleterious effects of crystalloid, is not compelling. The notion that the management of diabetic emergencies should be directed at the prevention of cerebral or pulmonary edema must, like the existence of the Loch Ness monster itself, be judged "not proven", in the tradition of Scottish jurisprudence. There is at present no role for the use of colloid in the usual patient with DKA or HNKC. There may be a role for the use of albumin in the occasional patient with DKA or HNKC who is in shock. This has not been studied. Crystalloid remains the treatment of choice for the correction of the fluid and electrolyte deficits in the usual patient with DKA or HNKC.

\section{References}

1. Schade DS, Eaton RP, Alberti KGMM, Johnston DG (1981) Diabetic coma, ketoacidotic and hyperosmolar. University of New Mexico Press, Albuquerque

2. Carroll P, Matz R (1983) Uncontrolled diabetes mellitus in adults: experience in treating diabetic ketoacidosis and hyperosmolar nonketotic coma with low-dose insulin and a uniform treatment regimen. Diabetes Care 6:579
3. Axelrod L (1982) Crystalloid infusions in diabetes. Lancet 2:548

4. Fein IA, Rackow EC, Sprung CL, Grodman R (1982) Relation of colloid osmotic pressure to arterial hypoxemia and cerebral edema during crystalloid volume loading of patients with diabetic ketoacidosis. Ann Intern Med 96:570

5. Krane EJ, Rockoff MA, Wallman JK, Wolfsdorf JI (1985) Subclinical brain swelling in children during treatment of diabetic ketoacidosis. N Engl J Med 312:1147

6. Winegrad AI, Kern EFO, Simmons DA (1985) Cerebral edema in diabetic ketoacidosis. N Engl J Med 312:1184

7. Carroll P, Matz R (1982) Adult respiratory distress syndrome complicating severely uncontrolled diabetes mellitus: report of nine cases and a review of the literature. Diabetes Care 5:574

8. Leonard RCF, Asplin C, McCormick CV, Hockaday TDR (1983) Acute respiratory distress in diabetic ketoacidosis: possible contribution of low colloid osmotic pressure. $\mathrm{Br}$ Med $\mathrm{J}$ 286:760

9. Powner D, Snyder JV, Grenvik A (1975) Altered pulmonary capillary permeability complicating recovery from diabetic ketoacidosis. Chest 68:253

10. Warshaw AL, Lesser PB, Rie M, Cullen DJ (1975) The pathogenesis of pulmonary edema in acute pancreatitis. Ann Surg 182:505

11. Lucas CE, Ledgerwood AM, Higgins RF, Weaver DW (1980) Impaired pulmonary function after albumin resuscitation from shock. J Trauma 20:446

12. Shires GT, III, Peitzman AB, Albert SA, Illner H, Silane MF, Perry MO, Shires GT (1983) Response of extravascular lung water to intraoperative fluids. Ann Surg 197:515

13. Virgilio RW, Rice CL, Smith DE, James DR, Zarins CK, Hobelmann CF, Peters RM (1979) Crystalloid vs. colloid resuscitation: Is one better? A randomized clinical study. Surgery 85:129

14. Lowe RJ, Moss GS, Jilek J, Levine HD (1977) Crystalloid vs colloid in the etiology of pulmonary failure after trauma: a randomized trial in man. Surgery $81: 676$

15. Metildi LA, Shackford SR, Virgilio RW, Peters RM (1984) Crystalloid versus colloid in fluid resuscitation of patients with severe pulmonary insufficiency. Surg Gynecol Obstet 158:207

16. Goodwin CW, Dorethy J, Lam V, Pruitt BA, Jr (1983) Randomized trial of efficacy of crystalloid and colloid resuscitation of hemodynamic response and lung water following thermal injury. Ann Surg 197:520

\author{
Dr. Lloyd Axelrod \\ The Diabetes Unit \\ Massachusetts General Hospital \\ Boston, MA 02114 \\ USA
}

